\title{
Analysis of Nonlinear Dust-Acoustic Shock Waves in an Unmagnetized Dusty Plasma with $q$-Nonextensive Electrons Where Dust Is Arbitrarily Charged Fluid
}

\author{
G. Mandal*, N. Y. Tanisha \\ Department of Electronics and Communication Engineering, East West University, Dhaka, Bangladesh \\ Email: ${ }^{*}$ gdmandal@ewubd.edu
}

Received 23 August 2014

\begin{abstract}
The nonlinear propagation of dust-acoustic (DA) shock waves in three-component unmagnetized dusty plasma consisting of nonextensive electrons, Maxwellian ions and arbitrarily charged mobile dust grain has been investigated. It is found that the presence of $q$-nonextensive electrons and ions can change the nonlinear behavior of shock wave. The standard reductive perturbation method is employed to study the basic features (phase speed, amplitude and width) of DA shock waves (DASWs) which are significantly modified by the presence of Maxwellian ions and nonextensive electrons. The present investigation can be very effective for understanding the nonlinear characteristics of the DASWs in space and laboratory dusty plasmas.
\end{abstract}

\section{Keywords}

Dusty Plasma, Nonextensive Electrons, Shock Waves, Solitary Waves

\section{Introduction}

The wave propagation in dusty plasmas has received much attention in the recent years because of its vital role in understanding different types of collective processes in space plasma environments, namely, lower and upper mesossphere, cometary tails, planetary rings, planetary magnetosphere, interplanetary spaces, interstellar media, etc. [1] [2]. The presence of charged dust grains in a plasma modifies the existing plasma wave spectra as well as introduces a number of novel eigen modes [viz. dust-ion acoustic (DIA) waves [3], dust-acoustic (DA) waves [4], dust lattice waves, etc.]. The dust grains acquire a negative charge by the collection of electrons [5] [6] because the thermal speed of the electrons is much higher than that of the ions. On the other hand, dust grains may become positively charged also due to a variety of processes including photo-electron emission by UV photons, thermoionic emission induced by radiative heating [5] [7], secondary electron production, etc. However, Shahmansouri and Tribeche have considered [8] the effects of arbitrary amplitude DA solitary waves in an unmagne-

\footnotetext{
"Corresponding author.
} 
tized, collisionless dusty plasma with electrons and ions featuring Tsallis distribution. Thus, arbitrarily (negatively or positively) charged dust is found to exist in most space and a laboratory dusty plasma system [5].

Over the last few decades, a great deal of attention has been paid to a nonextensive generalization of the Boltzmann-Gibbs-Shannon (BGS) entropy, first proposed by Tsallis [9]. The effects of nonextensivity of plasma constituents are quite common in astrophysical and cosmological scenarios like stellar polytropes [10], hadronic matter and quark-gluon plasma [11], protoneutron stars [12], collisionless thermal plasma [13] [14], etc. as well as its laboratory applications in nanomaterials, microdevices, and microstructures, etc. The nonextensive behavior of electrons and ions [13] (characterized by a parameter $q$ ) has been successfully employed in plasma physics. It is important to note that in case of $q<-1$, the $q$-distribution is not normalizable [2]. The strength of nonextensivity, $q$ varies as $-1<q<1$ [2]. Furthermore, for $q \geq 1$ [2] [15], the distribution function exhibits Maxwell-Boltzmann velocity distribution. On the other hand, negative ions are found to be an extra component (which may occur naturally or may be injected form external sources) in most space [16] or laboratory dusty plasmas [3]. The presence of those negative ions significantly modifies the characteristic features of the nonlinear propagation of waves in a dusty plasma system [17] [18].

Recently, Shahmansouri and Tribeche [19] have studied the DA shock waves in a charged varying dusty plasma with nonextensive ions as well as electrons. Therefore, in this paper, we have presented a more generalized theoretical investigation on the properties of DA shock waves (DASWs) in a multi-species dusty plasma system consisting of arbitrarily charged cold mobile dust fluid, positively charged ions following Maxwellian distributions, and nonextensive electrons. We are particularly interested to observe the effect of number density, nonextensive electrons, and dust kinematic viscosity on the DASWs in the dusty plasma system under consideration. We have shown here that the variations of relative ion number densities and temperature-ratio (with respect to electrons) are the sources of dissipation and are responsible for the formation of DASWs.

The manuscript is organized as follows. The governing equations are provided in Section 2. The Burgers equation is derived in Section 3. The numerical solution of Burgers equation is presented in Section 4. Finally, a brief discussion is given in Section 5.

\section{Governing Equations}

We consider the nonlinear propagation of the DA waves in an unmagnetized dusty plasma system consisting of arbitrarily charged mobile dust, nonextensive distributed electrons, and Maxwellian distributed ions. Thus, at equilibrium we have $n_{i 10}=n_{e 0}-j z_{d} n_{d 0}$, where $n_{i 10}$ is the density of ions at equilibrium, $z_{d}$ is the number of electrons residing onto the dust grain surface, $n_{e 0}\left(n_{d 0}\right)$ is the density of the electrons (dust particles) at equilibrium, and $j= \pm 1$ (+ for positively and-for negatively charged dust). The nonlinear dynamics of these lowfrequency electrostatic DA waves in such a dusty plasma system, whose phase speed is much smaller than the electron and ion thermal speeds but greater than the dust thermal speed, is governed by

$$
\begin{gathered}
\frac{\partial n_{d}}{\partial t}+\frac{\partial}{\partial x}\left(n_{d} u_{d}\right)=0 \\
\frac{\partial u_{d}}{\partial t}+u_{d} \frac{\partial u_{d}}{\partial x}=-j \frac{\partial \phi}{\partial x}+\eta \frac{\partial^{2} u_{d}}{\partial x^{2}} \\
\frac{\partial^{2} \phi}{\partial x^{2}}=\mu\left\{1+(q-1) \sigma_{1} \phi\right\}^{\frac{(q+1)}{2(q-1)}}-\mu_{i 1} \mathrm{e}^{-\phi}-j n_{d}
\end{gathered}
$$

where $n_{d}$ is the dust particle number density normalized by its equilibrium value $n_{d 0}, u_{d}$ is the dust fluid speed normalized by $C_{d}=\left(z_{d} k_{B} T_{i 1} / m_{d}\right)^{1 / 2}, \eta$ is the co-efficient of viscosity normalized by $\left(m_{d} n_{d 0} \omega_{p d} \lambda_{D m}^{2}\right)$, $\phi$ is the electrostatic wave potential normalized by $T_{i 1} / \mathrm{e}, \rho$ is the normalized surface charge density, $\sigma_{1}=T_{i 1} / T_{e}$, $\mu_{i 1}=n_{i 10} / z_{d} n_{d 0}$, and $\mu=n_{e 0} / z_{d} n_{d 0}=\mu_{i 1}+j$ It is also noted that $k_{B}$ is the Boltzmann constant, and $\mathrm{e}$ is the magnitude of the electron charge. The time variable $t$ is normalized by $\omega_{p d}^{-1}=\left(m_{d} / 4 \pi n_{d 0} z_{d}^{2} \mathrm{e}^{2}\right)^{1 / 2}$ and the space variable $x$ is normalized by $\lambda_{D m}=\left(T_{i 1} / 4 \pi n_{d 0} z_{d} \mathrm{e}^{2}\right)^{1 / 2}$. Consequently, the number densities of Maxwellian ions $n_{i 1}$ and nonextensive electrons $n_{e}$ [14], are given, respectively as 


$$
\begin{gathered}
n_{i 1}=n_{i 10}\left[\mathrm{e}^{-\mathrm{e} \phi / T_{i 1}}\right] \\
n_{e}=n_{e 0}\left[1+(q-1) \frac{\mathrm{e} \phi}{T_{i 1}}\right]^{\frac{q+1}{2(q-1)}}
\end{gathered}
$$

\section{Derivation of Burgers Equation}

To derive the Burgers equation (BE), we introduce the stretched coordinates [20] [21] as

$$
\begin{gathered}
\varsigma=\varepsilon\left(x-V_{p} t\right) \\
\tau=\varepsilon^{2} t
\end{gathered}
$$

where $V_{p}$ is the phase speed of the DA waves, and $\varepsilon$ is a smallness parameter measuring the weakness of the dispersion $(0<\varepsilon<1)$. We then expand $n_{d}, u_{d}$, and $\phi$ in power series of $\varepsilon$ :

$$
\begin{gathered}
n_{d}=1+\varepsilon n_{d}^{(1)}+\varepsilon^{2} n_{d}^{(2)}+\varepsilon^{3} n_{d}^{(3)}+\cdots \\
u_{d}=1+\varepsilon u_{d}^{(1)}+\varepsilon^{2} u_{d}^{(2)}+\varepsilon^{3} u_{d}^{(3)}+\cdots \\
\phi=\varepsilon \phi^{(1)}+\varepsilon^{2} \phi^{(2)}+\varepsilon^{3} \phi^{(3)}+\cdots
\end{gathered}
$$

and develop equations in various powers of $\varepsilon$. To the lowest order in $\varepsilon$, Equations (8)-(10) give

$$
\begin{gathered}
n_{d}^{(1)}=\frac{j}{V_{p}^{2}} \phi^{(1)} \\
u_{d}^{(1)}=\frac{j}{V_{p}} \phi^{(1)} \\
V_{p}=\frac{1}{\sqrt{\frac{1}{2} \mu \sigma_{1}(1+q)+\mu_{i 1}}}
\end{gathered}
$$

Equation (13) describes the phase speed of DA waves regarding the dusty plasma under consideration. To the next higher order of $\varepsilon$, we obtain a set of equations, which, after using Equations (11)-(13), can be simplified as

$$
\begin{gathered}
\frac{\partial n_{d}^{(1)}}{\partial \tau}-V_{p} \frac{\partial n_{d}^{(2)}}{\partial \varsigma}+\frac{\partial u_{d}^{(2)}}{\partial \varsigma}+\frac{\partial\left(n_{d}^{(1)} u_{d}^{(1)}\right)}{\partial \varsigma}=0 \\
\frac{\partial u_{d}^{(1)}}{\partial \tau}-V_{p} \frac{\partial u_{d}^{(2)}}{\partial \varsigma}+u_{d}^{(1)} \frac{\partial u_{d}^{(1)}}{\partial \varsigma}+j \frac{\partial \phi^{(2)}}{\partial \varsigma}=\eta \frac{\partial^{2} u_{d}^{(1)}}{\partial \varsigma^{2}} \\
\frac{1}{4} \mu \sigma_{1}^{2}(5+q)(3-q)\left\{\phi^{(1)}\right\}^{2}+\frac{1}{2} \mu \sigma_{1}(1+q) \phi^{(2)}-\mu_{i 1} \phi^{(2)}-\frac{1}{2} \mu_{i 1}\left\{\phi^{(1)}\right\}^{2}-j n_{d}^{(2)}=0
\end{gathered}
$$

Now combining Equations (14)-(16), we obtain an equation of the form:

$$
\frac{\partial \phi}{\partial \tau}+A \phi \frac{\partial \phi}{\partial \varsigma}=C \frac{\partial^{2} \phi}{\partial \varsigma^{2}}
$$

where

$$
\begin{gathered}
A=\frac{V_{p}^{3}}{2}\left[\mu_{i 1}-\frac{1}{4} \sigma_{1}^{2} \mu(1+q)(3-q)+\frac{3 j}{V_{p}^{4}}\right] \\
C=\frac{\eta}{2}
\end{gathered}
$$


Equation (17) is known as Burger's equation. The stationary localized solution of the BE is given by

$$
\psi=\psi_{m}\left[1-\tanh \left(\frac{\varsigma}{\delta}\right)\right]
$$

where $\psi=\phi, \delta=2 C / U_{0}$ is the width, and $\psi_{m}=U_{0} / A$ is the amplitude of the shock profile.

\section{Numerical Analysis}

It is obvious from Equation (20), for $A>(<) 0$, the dusty plasma supports rarefactive (compressive) DASWs which are associated with a positive (negative) potential, and no shock waves exist at $A=0$ and $A \sim 0$. We note that the nonlinearity coefficient $A$ is a function of $\mu_{i 1}, \sigma_{1}$ and $q$ for the model under consideration in this manuscript. So, to find the parametric regimes correspond to $A=0$, we consider a certain ranges of the dusty plasma parameters $\sigma_{1}=0.1 \sim 0.3$ [2], for $j=1$ and $q=-0.3 \sim 0.7$ [2] [15] are used in this numerical analysis. It is clear that $\psi=\infty$ at $\mu_{i 1}=\mu_{p}\left(\mu_{n}\right)$ for positive dust (for negative dust). This means that the existence of the small amplitude DASWs with a positive potential for $\mu_{i 1}>\mu_{p}\left(\mu_{i 1}>\mu_{n}\right)$, and with a negative potential for $\mu_{i 1}<\mu_{p}\left(\mu_{i 1}<\mu_{n}\right)$. However, this Burgers equation is not valid when $\mu_{i 1} \sim \mu_{p}$ (or $\mu_{i 1} \sim \mu_{n}$ ). This is because the $\mu_{i 1} \sim \mu_{p}$ (for $j=1$ ) and $\mu_{i 1} \sim \mu_{n}$ (for $j=-1$ ) give rise to infinitely large amplitude structures which break down the validity of the reductive perturbation method applied for deriving the Burgers equation. This implies that DASWs are formed for the values far above or below the critical values.

\section{Discussion}

We have studied that the nonlinear propagation of DASWs in an unmagnetized dusty plasma consisting of arbitrarily charged mobile dust fluid, positive ions following Maxwellian distributions, and electrons following $q$ distribution. The propagation of the small amplitude nonlinear DASWs in dusty plasmas has been considered for analyzing the solution of the Burgers' equation. The Burgers' equation has been derived by using the reductive perturbation technique. The basic properties (i.e. polarity, amplitude, width, etc.) of the DASWs in dusty plasmas are found to be significantly modified by the number densities of ions (viz. $n_{i 1}$ ), nonextensive parameter $q$, and temperatures of electrons and ions $\left(T_{e}\right.$, and $T_{i 1}$ ), respectively. The important findings of our current analysis can be summarized as follows:

1. The dusty plasmas under consideration support finite-amplitude shock structures whose basic features (polarity, amplitude, width, speed, etc.) strongly depend on different plasma parameters, particularly, $T_{i 1}, n_{i 1}$, and $q$.

2. The widths of the DASWs in dusty plasmas with positively charged dust increase with increasing $\eta$ as shown in Figure 1. In addition, it can also be said that with the increase of dissipation, the DASWs become smoother and weaker. We have also observed the effect of speed on the propagation of DASWs in Figure 1; the amplitudes (widths) of the shock structures increase (decrease) with the increasing of $U_{0}$.

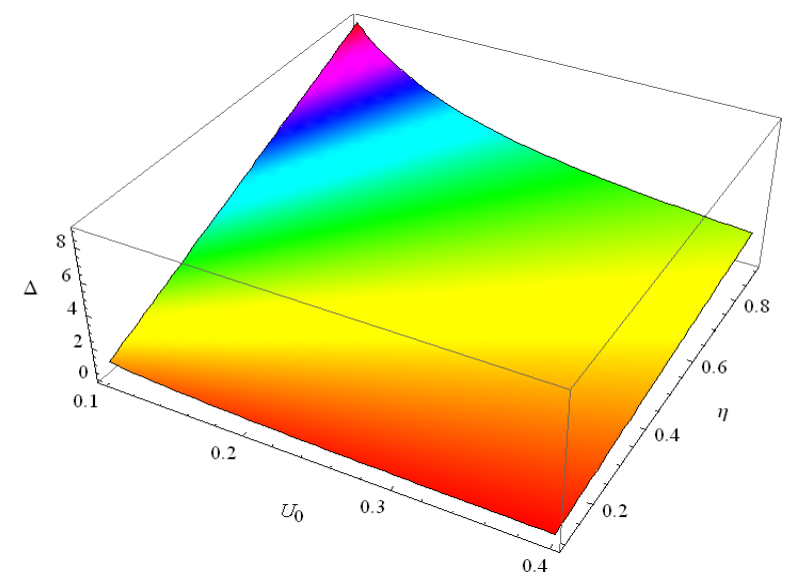

Figure 1. Showing the variation of width $(\Delta)$ of shock structure with $U_{0}$ and $\eta$ for $\sigma_{1}=0.2, q=0.3$ and $j=1$. 
3. For positively charged dust $(j=1)$, the DASWs with positive potential are formed at $\mu_{i 1}>\mu_{p}$ as shown in Figure 2. Again, for negatively charged dust $(j=-1)$, the DASWs with negative potential are formed at $\mu_{i 1}>\mu_{n}$ as presented in Figure 3.

4. For $j=1$, the magnitude of the amplitude of positive potential DASWs decreases with the increasing of temperature-ratio of light ions, $\sigma_{1}$ as shown in Figure 4. For $j=-1$ also, the magnitude of the amplitude of negative potential DASWs decreases with the increasing of $\sigma_{1}$ as presented in Figure 5.

5. The effects of nonextensive parameter $(q)$ on the DASWs in the considered dusty plasma model are presented in Figure 6 for $j=1$ and Figure 7 for $j=-1$.

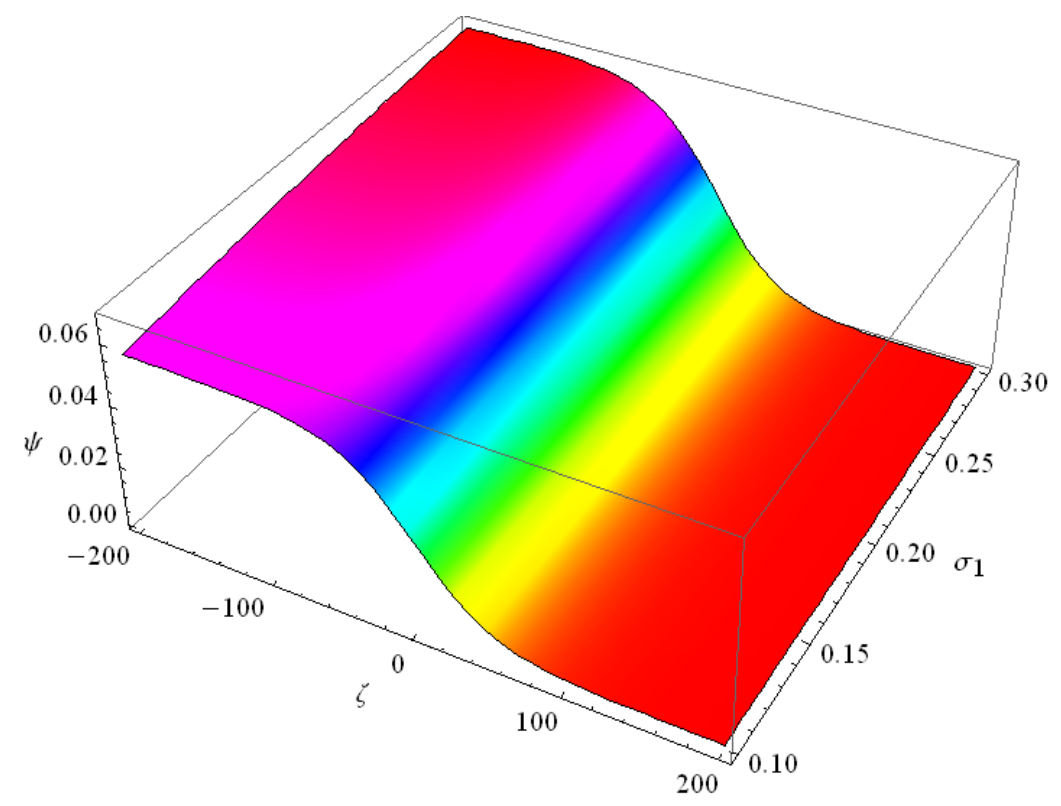

Figure 2. Positive shock structure is shown with the variation of $\sigma_{1}$ (where $\mu_{i 1}>\mu_{p}$ for $j=1$ ) on DASWs for $q=0.3$, and $\eta=0.5$.

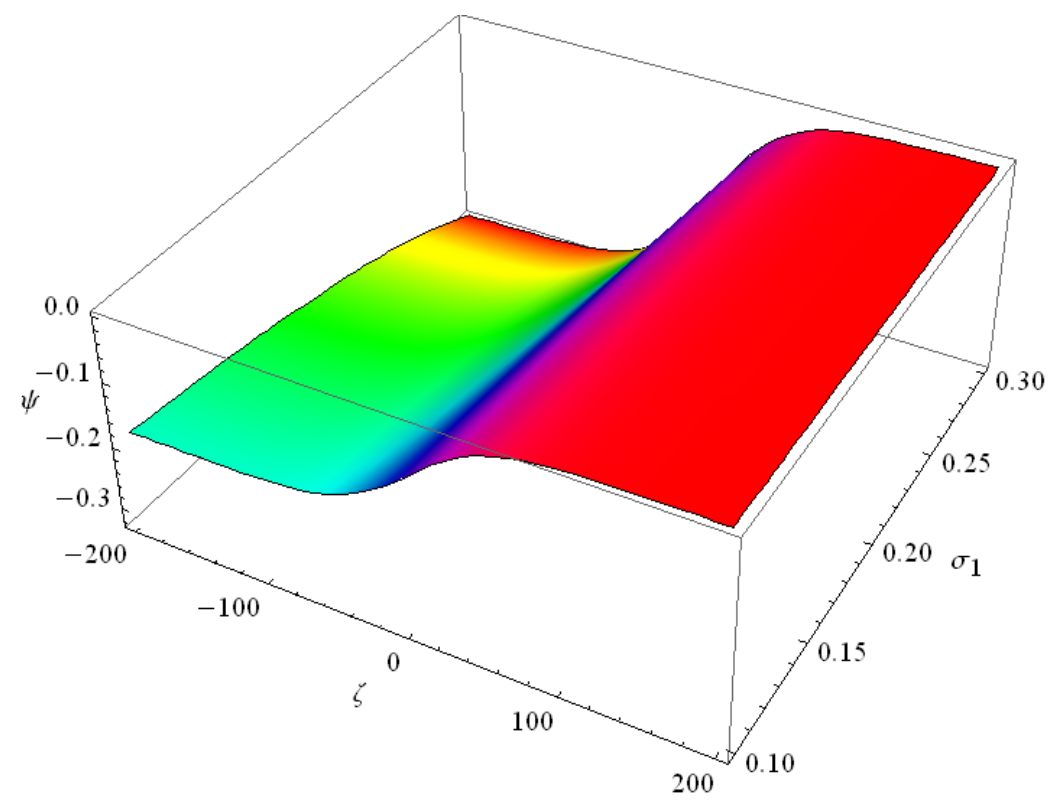

Figure 3. Negative shock structure is shown with the variation of $\sigma_{1}$ (where $\mu_{i 1}>\mu_{n}$ for $j=-1$ ) on DASWs for $q=0.3$, and $\eta=0.5$. 


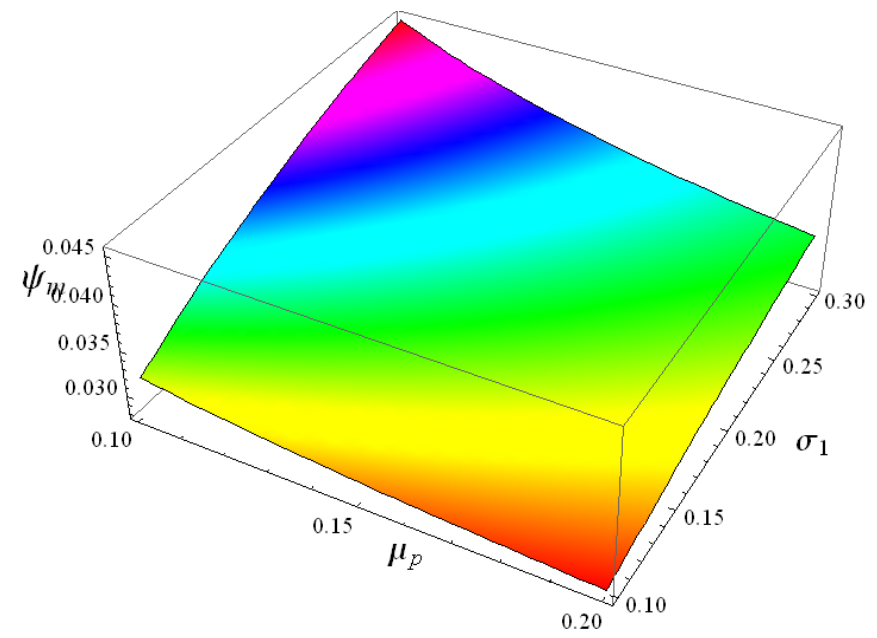

Figure 4. The variation of amplitude $\psi_{m}$ with $\mu_{p}$ and $\sigma_{1}$ of DASWs in the dusty plasma system (where $\mu_{i 1}>\mu_{p}$ for $j=1$ ) where $q=0.3$, and $\eta=0.5$.

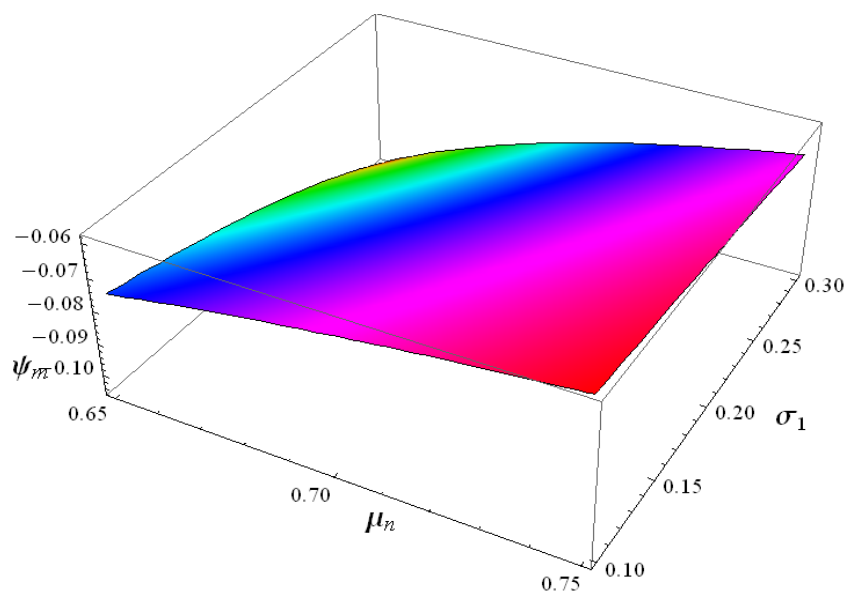

Figure 5. The variation of amplitude $\psi_{m}$ with $\mu_{n}$ and $\sigma_{1}$ of DASWs in the dusty plasma system (where $\mu_{i 1}>\mu_{n}$ for $j=1$ ) where $q=0.3$, and $\eta=0.5$.

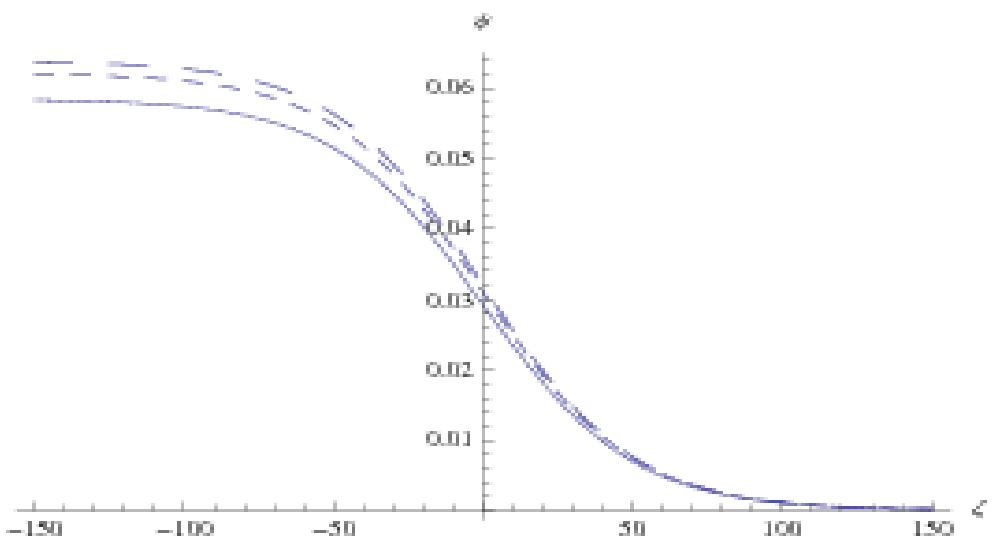

Figure 6. The variation of the solution $\psi$ of the Berger's Equation with $\eta$ (where $\mu_{i 1}<\mu_{n}$ for $j=1$ ) for $\sigma_{1}=0.2, q=-0.3$ (solid curve), $q=0.1$ (dotted curve), $q=0.7$ (long dashed curve). 


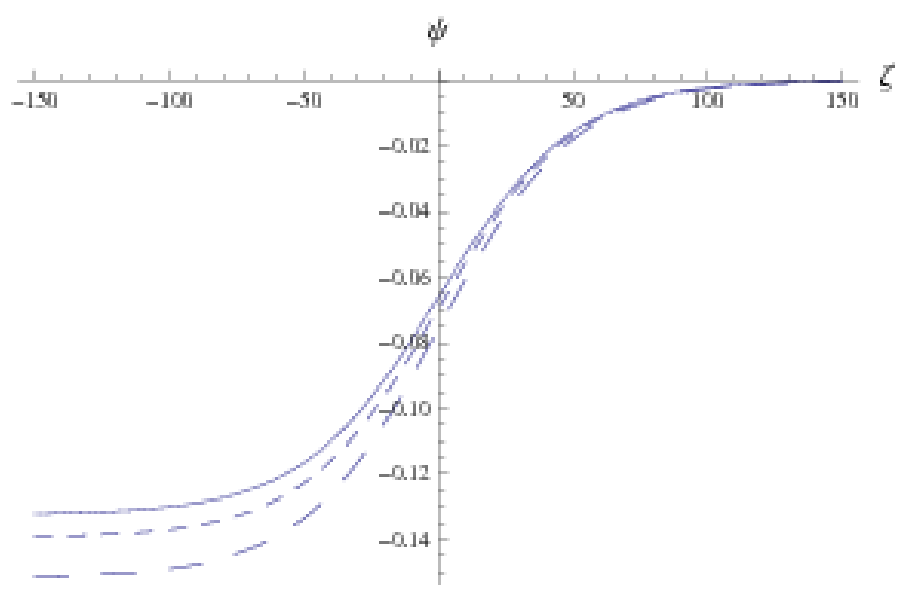

Figure 7. The variation of the solution $\psi$ of the Berger's Equation with $\eta$ (where $\mu_{i 1}<\mu_{n}$ for $j=-1$ ) for $\sigma_{1}=0.2, q=-0.3$ (solid curve), $q=0.1$ (dotted curve), $q=0.7$ (long dashed curve).

It can be noted here that the effects of the external magnetic field, nonplanar solitary [5] and shock structures, and their instabilities [3] are also problems of great importance, but are beyond the scope of our present investigation. Further, we stress that our present investigation should be very useful and effective in understanding the salient features of localized DASWs in space plasmas [3] [17].

\section{References}

[1] Mendis, D.A. and Rosenberg, M. (1994) Annu. Rev Astron. Astrophys., 32, 419. http://dx.doi.org/10.1146/annurev.aa.32.090194.002223

[2] Verheest, F. (2000) Waves in Dusty Space Plasmas. Kluwer Academic Press, Dordrecht. http://dx.doi.org/10.1007/978-94-010-9945-5

[3] Rosenberg, M. and Merlino, R.L. (2007) Planet Space Sci., 55, 1464. http://dx.doi.org/10.1016/j.pss.2007.04.012

[4] Rao, N.N., Shukla, P.K. and Yu, M.Y. (1990) Planet. Space Sci., 38, 543. http://dx.doi.org/10.1016/0032-0633(90)90147-I

[5] Shukla, P.K. and Mamun, A.A. (2002) Introduction to Dusty Plasma Physics. Institute of Physics Publishing Ltd., Bristol. http://dx.doi.org/10.1887/075030653X

[6] Rosenberg, M. and Mendis, D.A. (1995) IEEE Trans. Plasma Sci., 23, 177. http://dx.doi.org/10.1109/27.376584

[7] Goertz, C.K. (1989) Rev. Geophys., 27, 271. http://dx.doi.org/10.1029/RG027i002p00271

[8] Shahmansouri, M. and Tribeche, M. (2013) Astrophys. Space Sci., 344, 99-104. http://dx.doi.org/10.1007/s10509-012-1296-y

[9] Tsallis, C. (1988) J. Stat. Phys., 52, 479. http://dx.doi.org/10.1007/BF01016429

[10] Plastino, A.R. and Plastino, A. (1993) Phys. Lett. A, 174, 384. http://dx.doi.org/10.1016/0375-9601(93)90195-6

[11] Gervino, G., Lavagno, A. and Pigato, D. (2012) Central Euro. J. Phys., 10, 594. http://dx.doi.org/10.2478/s11534-011-0123-3

[12] Lavagno, A. and Pigato, D. (2011) Euro. Phys. J. A, 47, 52. http://dx.doi.org/10.1140/epja/i2011-11052-1

[13] Lima, J.A.S., Silva, R. and Santos, J. (2000) Phys. Rev. E, 61, 3260. http://dx.doi.org/10.1103/PhysRevE.61.3260

[14] Pakzad, H.R. (2011) Phys. Scr., 83, Article ID: 015505.

[15] Tribeche, M. and Merriche, A. (2011) Phys. Plasmas, 18, Article ID: 034502.

[16] D’Angelo, N. (2004) J. Phys. D, 37, 860. http://dx.doi.org/10.1088/0022-3727/37/6/009

[17] Mamun, A.A., Shukla, P.K. and Eliasson, B. (2009) Phys. Plasmas, 16, Article ID: 1145031. http://dx.doi.org/10.1063/1.3261840

[18] Paul, A., Mandal, G., Mamun, A.A. and Amin, M.R. (2011) IEEE Trans. Plasma Sci., 39, 1254. http://dx.doi.org/10.1109/TPS.2011.2120627 
[19] Shahmansouri, M. and Tribeche, M. (2013) Astrophys. Space Sci., 346, 165-170. http://dx.doi.org/10.1007/s10509-013-1430-5

[20] Schamel, H. (1972) J. Plasma Phys., 14, 905. http://dx.doi.org/10.1088/0032-1028/14/10/002

[21] Schamel, H. (1973) J. Plasma Phys., 9, 377. http://dx.doi.org/10.1017/S002237780000756X 